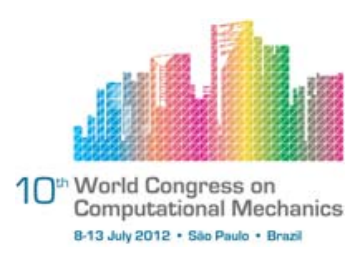

\title{
ITM-Based FSI-Models for Room Acoustical Simulations
}

\author{
M. Buchschmid ${ }^{1}$, G. Müller ${ }^{1}$, M. Pospiech ${ }^{2}$ \\ ${ }^{1}$ Chair of Structural Mechanics, Technische Universität München \\ (martin.buchschmid@bv.tum.de) \\ ${ }^{2}$ Chair of Numerical Analysis, Technische Universität München
}

\begin{abstract}
In many different fields of engineering, like automotive industry or civil engineering, room acoustical tasks are of interest. Sound fields have to be predicted in order to design the acoustic cavity by placing acoustic elements like reflectors or absorbers (passive absorbers or plate resonators) into the room for example. Therefore models for the Fluid Structure Interaction (FSI) are used, where passive absorbers or plate resonators can be considered. For simulations of the spatial resolution of the sound field within acoustic cavities very often techniques based on Finite Element formulations are used. In order to reduce the number of degrees of freedom and therefore the numerical effort, a model reduction method, based on a Component Mode Synthesis (CMS), is applied in this contribution. The advantage is related to the fact, that the modal analysis is done only once for the rigid walled cavity, which is modeled with Spectral Finite Elements (SFEM). The cavity boundary conditions, e.g. compound absorbers made of homogenous plates and porous foams, are modeled using Integral Transform Methods (ITM). Therefore the differential equations of motion are established for the individual components, where the Lamé Equation is used for homogenous and the Theory of Porous Media (TPM) for porous materials. These equations are solved in the wavenumber-frequency domain after applying a Fourier Transformation. The results (wavenumber dependent impedances) for the absorptive structure are coupled with the acoustic cavity adding interface coupling modes for the fluid and applying Hamilton's principle, considering the velocity of both components to coincide as a constraint at the interface. The method is presented and models of the subsystems, the absorber and the fluid, are shown. Finally examples for the simulation of the coupled structure are presented.
\end{abstract}

Keywords: Room Acoustics, Fluid Structure Interaction, Component Mode Synthesis, Integral Transform Methods.

\section{INTRODUCTION}

Due to increasing requirements of comfort, acoustic design has become more important during the last years, especially in the field of civil engineering and automotive design. 
The sound field within rooms or vehicles has to be predicted and then modified in an acoustic optimization process in order to reach an optimal result for the specific use.

The calculation of the sound pressure level inside of acoustic cavities is usually done with the help of the Statistical Energy Analysis (SEA). This method is robust for systems with a high modal density and it is based on an averaging over frequency bands, points of excitation and points of observation. However, its performance is limited if a description of the spatial resolution of the response is necessary and if the influence of boundary conditions has to be described in detail.

The acoustical improvement of rooms or vehicles is done by placing elements like reflectors or absorbers (e.g. passive absorbers or plate resonators) into the sound field. Therefore a robust method for the phase correct modeling of interior sound fields with sufficient spatial resolution is needed, where the absorptive behavior of the delimiting surfaces can be considered. Methods, based on Finite Element formulations are used for this purpose, where the robustness can be enhanced by applying an averaging in the post-processing. This requires multiple calculations with slight changes in geometry or load data [5].

Finite Element models for absorptive boundary conditions in acoustical calculations lead to a huge number of degrees of freedom. In order to reduce this number of unknowns in [11] and [7] an impedance approach considering a plane wave of incidence is used. In [6], [3] and in the scope of the presented work a wavenumber dependent impedance is used for plate-like compound absorbers to introduce varying angles of incidence for the sound wave. The porous foam, which is part of compound absorbers, is modeled by the TPM [1].

An efficient way, especially to reduce the computational effort for the optimization of the position of these acoustic elements or for averaging techniques as mentioned above, is to simulate the coupled system (fluid-structure) in the frequency domain by applying a CMS.

\section{FLUID STRUCTURE INTERACTION}

The derivation of the FSI method is carried out in the frequency domain. Therefore only forces, harmonically oscillating in time, with the circular frequency of excitation $\Omega$ are considered. Consequently the steady state solution for both state variables pressure $p_{A}$ and sound velocity $\mathbf{v}_{A}$ is harmonic in time.

\subsection{Hamiltons Principle and Ritz Approach}

For the vibro-acoustical problem discussed in this contribution a description of Hamilton's principle, which is based on velocities, is applied. The structure is divided into substructures (see figure 1), where the acoustic fluid and the boundary conditions are defined as subsystems respectively. According to Hamilton's principle equilibrium is fulfilled by the velocity field, which meets the kinematic boundary conditions, the conditions at $t=t_{1}$ and $t=t_{2}$ and, in addition to that, satisfies

$$
\int_{t_{1}}^{t_{2}} \delta\left(L_{A}(t)+L_{B C}(t, Z)+\mathbf{R}^{T} \boldsymbol{\lambda}(t)\right)+\delta W_{B C}^{n c}(t, Z)+\delta W_{L o a d}^{n c}(t) d t=0 .
$$




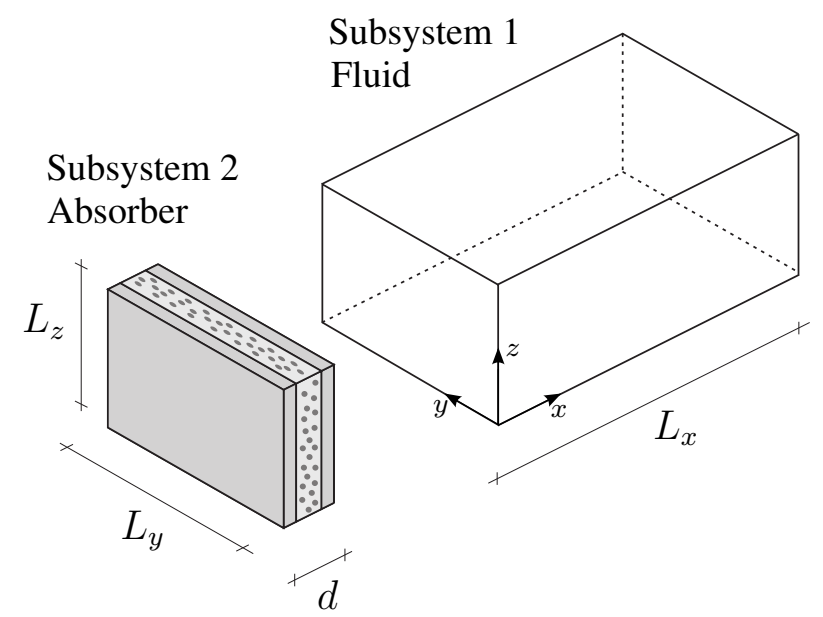

Figure 1. Subsystem definition

The Lagrangian function $L_{A}$ for the acoustic fluid results from the kinetic energy $T_{A}$ and the potential energy $U_{A}$

$$
L_{A}(t)=T_{A}(t)-U_{A}(t),
$$

where the energies are computed out of

$$
T_{A}(t)=\frac{\rho_{A}}{2} \int_{V}\left|\mathbf{v}_{A}(\mathbf{x}, t)\right|^{2} d V \quad \text { and } \quad U_{A}(t)=\frac{1}{2 \rho_{A} c_{A}^{2}} \int_{V}\left|p_{A}(\mathbf{x}, t)\right|^{2} d V
$$

respectively. The harmonically oscillating load is considered in Hamilton's Principle as a non conservative force by its virtual work $\delta W_{\text {Load }}^{n c}$

$$
\delta W_{\text {Load }}^{n c}(t)=\int_{A_{\text {Load }}} p_{\text {Load }}(\mathbf{x}, t) \mathbf{n}_{\text {Load }}(\mathbf{x}) \delta \mathbf{w}(\mathbf{x}, t) d A .
$$

The formulation of $L_{B C}$ and $\delta W_{B C}^{n c}$ will be given for a wavenumber-dependent impedance in Chapter 3. In the scope of a Ritz approach, using the ansatz functions, which will be defined in section 2.2 a linear equation system is obtained to compute the unknown coefficients.

\subsection{Component mode Synthesis}

The Component Mode Synthesis (CMS) is a substructuring technique for large coupled problems, which was introduced by Hurty $[9,10]$ to reduce the number of unknowns while keeping the physical characteristics of the structure. In contrast to Hurty, the CMS is used based on a modal description in the scope of this method. In order to model arbitrary geometries for the acoustic fluid the numerical approach developed in [12], which is based on the Spectral Finite Element Method (SFEM) is used. In the frame of the CMS the superscript $N$ stands for normal modes and the superscript $C$ for coupling modes. Normal modes are the eigenmodes of the air volume enclosed by totally reflecting boundaries and coupling modes are additionally introduced to provide the coupling to other boundary conditions, like a deformable structure, an absorber or an open interface to another acoustic volume. For the 
velocity $\mathbf{v}_{A}$ in the acoustic fluid the approach (4) is applied.

$$
\mathbf{v}_{A}(\mathbf{x}, t)=\sum_{m} \mathbf{v}_{m}^{N}(\mathbf{x})\left(\mathcal{A}_{m} e^{i \Omega t}+\overline{\mathcal{A}}_{m} e^{-i \Omega t}\right)+\sum_{n} \mathbf{v}_{n}^{C}(\mathbf{x})\left(\mathcal{B}_{n} e^{i \Omega t}+\overline{\mathcal{B}}_{n} e^{-i \Omega t}\right)
$$

Assuming an acoustic fluid, the irrotational behavior of the sound velocity allows the use of a velocity potential $\Phi_{A}(\mathbf{x}) e^{i \Omega t}$.

$$
\mathbf{v}_{A}(\mathbf{x}, t)=\operatorname{grad} \Phi_{A}(\mathbf{x}, t)
$$

Considering the steady state problem after applying a Fourier-transformation from the timeto the frequency-domain, the velocity potential solves the Helmholtz equation (5), where $c_{A}$ denotes the constant speed of sound.

$$
\Delta \hat{\Phi}_{A}(\mathbf{x}, \omega)+\frac{\omega^{2}}{c_{A}^{2}} \hat{\Phi}_{A}(\mathbf{x}, \omega)=0
$$

In the Fourier-transformed domain the velocity $\hat{\mathbf{v}}_{A}$ and the pressure $\hat{p}_{A}$ read as follows:

$$
\begin{gathered}
\hat{\mathbf{v}}_{A}(\mathbf{x}, \omega)=\operatorname{grad} \hat{\Phi}_{A}(\mathbf{x}, \omega) \\
\hat{p}_{A}(\mathbf{x}, \omega)=-\frac{\rho_{A} c_{A}^{2}}{i \omega} \operatorname{div} \hat{\mathbf{v}}_{A}(\mathbf{x}, \omega) \\
=-\frac{\rho_{A} c_{A}^{2}}{i \omega} \Delta \hat{\Phi}_{A}(\mathbf{x}, \omega)
\end{gathered}
$$

The normal modes for the acoustic fluid are defined in terms of the velocity potential $\hat{\Phi}^{N}$ assuming fixed interfaces, which means reflective wall conditions for all boundaries of the fluid:

$$
\operatorname{grad} \hat{\Phi}_{m}^{N}\left(\mathbf{x}, \omega_{m}\right) \cdot \mathbf{n}_{B C}=0
$$

The normal modes are supplemented by coupling modes in order to define a valid set of trial functions for (4). These coupling modes enable velocities perpendicular to the coupling interface. They fulfill the reflective boundary conditions at all surfaces of the room, except for the interface defined as $\mathbf{x}_{\mathrm{BC}}$, where modal trial functions $g\left(\mathbf{x}_{B C}\right)$ are prescribed.

$$
\operatorname{grad} \hat{\Phi}_{n}^{C}(\mathbf{x}, \Omega) \cdot \mathbf{n}_{B C}=g\left(\mathbf{x}_{B C}\right)
$$

In the following a rectangular geometry is considered for the absorptive boundary condition and $g\left(\mathbf{x}_{B C}\right)$ is expressed with $\psi_{n}(y, z)$ for each mode, where $y$ and $z$ mark the local coordinates in the reference coordinate system of the absorber. Considering the procedural method in the next steps, in especially the computation of the Lagrangian $L_{B C}$ for the absorber out of impedances, it is advantageous to express $\psi_{n}(y, z)$ with its Fourier Series.

$$
\hat{\psi}_{n}(y, z)=\sum_{r} \sum_{s} E_{n r s} e^{i\left(k_{y}(r) y+k_{z}(s) z\right)}
$$

Thus the trial function for at the interface is specified for an absorptive boundary condition as

$$
\hat{v}_{B C}(y, z, t)=\sum_{n} \hat{\psi}_{n}(y, z)\left(\mathcal{C}_{n} e^{i \Omega t}+\overline{\mathcal{C}}_{n} e^{-i \Omega t}\right)
$$


Carrying out the integration required in equation (1) over one period of the steady state vibration one obtains the Lagrangian $L_{B C}$ and the virtual work of the non conservative forces $\delta W_{B C}$. In this short essay we focus on trial functions, where due to reasons of orthogonality the off diagonal terms vanish.

$$
\begin{array}{r}
\int_{0}^{T} L_{B C} d t=\frac{T}{\Omega} L_{y} L_{z}\left[\sum_{n} \mathcal{C}_{n} \overline{\mathcal{C}}_{n} \sum_{r} \sum_{s} \operatorname{Im}(Z(r, s, \Omega))\left|E_{n r s}\right|^{2}\right] \\
\int_{0}^{T} \delta W_{B C} d t=-\frac{T}{i \Omega} L_{y} L_{z} \sum_{n}\left(\overline{\mathcal{C}}_{n} \delta \mathcal{C}_{n}-\mathcal{C}_{n} \delta \overline{\mathcal{C}}_{n}\right) \sum_{r} \sum_{s} \operatorname{Re}(Z(r, s, \Omega))\left|E_{n r s}\right|^{2}
\end{array}
$$

In case of sinusoidal functions also the Fourier approximation $\hat{\psi}_{n}(y, z)$ can be omitted. A detailed discussion as well as the expressions for a general definition of the trial functions are presented in [3]. With the imaginary part of the impedance $\operatorname{Im}(Z(r, s, \Omega))$ the flexible characteristics of the absorber can be modeled, as shown above. The absorptive characteristics are expressed by the real part of impedance $\operatorname{Re}(Z(r, s, \Omega))$.

\section{COMPOUND ABSORBERS}

In the next step these impedances $Z(r, s, \Omega)$, depending on the circular frequency of excitation $\Omega$ and the wavenumbers $k_{y}(r)$ and $k_{z}(s)$ in order to build up the dependency on the angle of incidence, are derived for compound absorbers containing porous layers.

\subsection{Classification}

Absorbers used in room acoustics can be classified in passive absorbers and plate- or Helmholtz resonators (figure 2). Passive absorbers consist of porous materials like foams, mineral wool or cellular glass. The sound waves enter the pores of the absorber and initiate a vibration of the air in the interconnected pores. The kinetic energy of the sound field is reduced due to the flow resistance within the porous absorber. In case of resonators either a

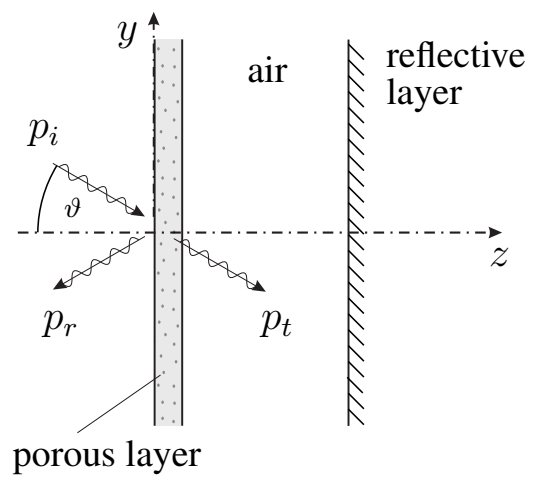

Passive Absorber

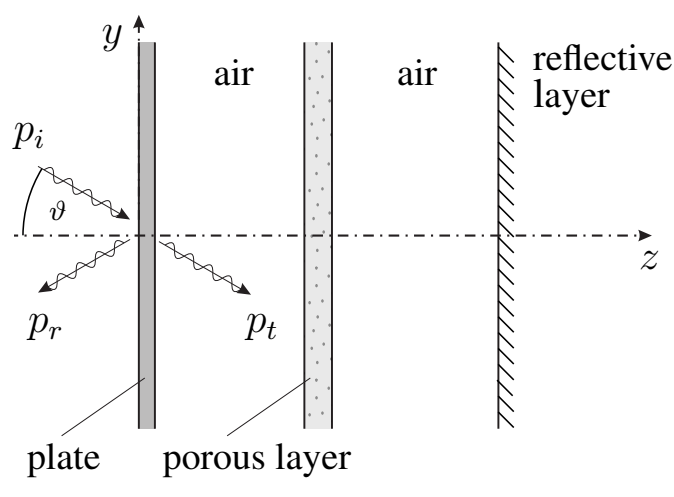

Plate Resonator

Figure 2. Classification of absorbers

flexible homogeneous plate or a Helmholtz resonator is excited to vibrations by sound waves. 
Energy is reduced due to internal damping, which can be increased by absorption in a porous layer, installed in the air cushion behind the vibrating plate, or inside the resonator. In order to describe these kinds of absorptive structures, a layered model, in which homogeneous material, porous material and air are combined, has to be used.

In the following sections the theory for modeling these structures and the solution of the differential equation system are presented. In contrast to the detailed derivation, which is published in $[3,4]$, a short review is given in the scope of this contribution. Finally the boundary conditions, which are needed to specify the coefficients of the fundamental system for compound structures, are listed.

\subsection{Material Description}

For modeling porous layers of an absorber the linear Theory of Porous Media (TPM) is applied. The porous medium, consisting of the constituents solid and gas (air) is modeled as a smeared volume with statistically distributed pores, whereas the solid phase spans the control space $[1,2]$. The porous solid is assumed to be much stiffer than the air. This results in a structural compressibility of a material, which consists of an incompressible porous solid and a compressible gas.

The volume fractions concept allows to identify the individual constituents in the smeared continuum. Volume fractions $n_{\alpha}$ refer the volume element $d v_{\alpha}$ of the constituent $\alpha$ to the volume element of the mixture $d v$.

$$
n_{\alpha}(\mathbf{x}, t)=\frac{d v_{\alpha}}{d v}
$$

Due to the assumption, that the pores are completely filled with gas, the constituents $\varphi_{\alpha}$ must fulfill the saturation condition (15), which reads:

$$
\sum n_{\alpha}=1
$$

The stress tensor of the solid component consists of a weighted pore pressure and the solid extra stresses. It is defined as

$$
\mathbf{T}_{S}=-n_{S} p \mathbf{I}+\tilde{\lambda}_{S}\left(\mathbf{E}_{S} \cdot \mathbf{I}\right) \mathbf{I}+2 \mu_{S} \mathbf{E}_{S}
$$

The stresses in the gas phase are expressed by a weighted pore pressure.

$$
\mathbf{T}_{G}=-n_{G} p \mathbf{I}
$$

The balance and conservation laws in mechanics have to be satisfied for each constituent $\varphi_{\alpha}$. Their interaction is considered by an interaction force $\hat{\mathbf{p}}_{\alpha}$, which depends on the on the seepage velocity between the solid- and the gas-phase as well as on the permeability constant $S_{G}$. The conservation of momentum results in

$$
\begin{array}{r}
-n_{S} \operatorname{grad} p+\left(\tilde{\lambda}_{S}+\mu_{S}\right) \operatorname{grad} \operatorname{div} \mathbf{u}_{S}+\mu_{S} \operatorname{div} \operatorname{grad} \mathbf{u}_{S}+S_{G}\left(\mathbf{v}_{G}-\mathbf{v}_{S}\right)=\rho_{S} \mathbf{a}_{S} \\
-n_{G} \operatorname{grad} p-S_{G}\left(\mathbf{v}_{G}-\mathbf{v}_{S}\right)=\rho_{G} \mathbf{a}_{G}
\end{array}
$$


where $\tilde{\lambda}_{S}$ and $\mu_{S}$ are the macroscopic Lamé constants. The conservation of mass finally reads

$$
n_{G} \frac{\partial \rho_{G R}}{\partial t}+\rho_{G R} n_{G} \operatorname{div}\left(\mathbf{v}_{G}\right)+\rho_{G R} n_{S} \operatorname{div}\left(\mathbf{v}_{S}\right)=0 .
$$

The layers, consisting of homogeneous, linear-elastic, isotropic material are described by the Lamé Equation. Assuming the external acceleration to be zero the equation is given in a cartesian reference frame by

$$
\left(\lambda_{H}+\mu_{H}\right) \operatorname{grad} \operatorname{div} \mathbf{u}_{H}+\mu_{H} \operatorname{div} \operatorname{grad} \mathbf{u}_{H}=\rho_{H} \mathbf{a}_{H} .
$$

The subscript $H$ denotes the homogeneous material in order to distinguish from the constituents of the porous medium in the following.

The homogeneous and porous layers, discussed in the previous part, interact within the model of the porous absorber with the acoustic fluid. The differential equation for the air is the wave equation.

$$
\Delta p_{A}-\frac{1}{c_{A}^{2}} \frac{\partial^{2} p_{A}}{\partial t^{2}}=0
$$

The subscript $A$ specifies the air.

The equations (18), (19), (20) and (21), specified above can be simplified expressing the displacement field with a scalar potential $\Phi$ and a vector potential $\Psi$ [8]. They are defined in equation (23) for the homogeneous material and in equations (24) and (25) for the porous material.

$$
\begin{aligned}
\mathbf{u}_{H} & =\operatorname{grad} \Phi_{H}+\operatorname{rot} \boldsymbol{\Psi}_{H} \\
\mathbf{u}_{S} & =\operatorname{grad} \Phi_{S}+\operatorname{rot} \boldsymbol{\Psi}_{S} \\
\mathbf{u}_{G} & =\operatorname{grad} \Phi_{G}+\operatorname{rot} \boldsymbol{\Psi}_{G}
\end{aligned}
$$

Using a Fourier transform into the wavenumber frequency domain the partial differential equations and systems of PDEs can be simplified to ordinary differential equations and systems of ODEs respectively. The time $t$ is transformed into the frequency domain $(t \multimap \Omega)$ because of a harmonic excitation. The spatial coordinates $x$ and $y$ are transformed into the wavenumber domain $\left(x \circ \longrightarrow k_{x}\right)$ and $\left(y \supset \longrightarrow k_{y}\right)$, assuming infinite dimensions in two directions.

The disadvantage of this transformation is the loss of the possibility to vary the parameters of the system in $x$ - and $y$-direction. However for the applications in acoustics, presented in this paper, especially for an application in room acoustics the definition of different layers in $z$-direction is sufficient (see figure 2).

The solution can be carried out in the transformed domain using an exponential approach as depicted in [3].

\subsection{Boundary Conditions and Equation System}

In order to specify the coefficients of the respective fundamental system the boundary conditions have to be evaluated for the individual compound absorber. These boundary conditions are sketched exemplarily for the interface between a homogeneous and a porous layer 


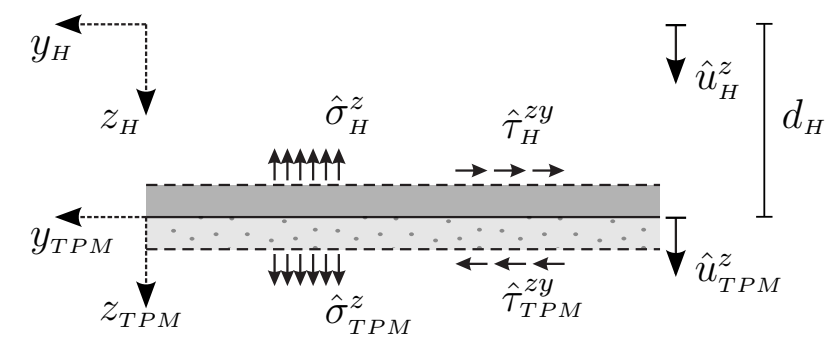

Figure 3. Interface between a homogeneous and a porous layer

in the following. The transformed quantities are marked with a hat and the normal vector $\mathbf{n}=\left[\begin{array}{lll}0 & 0 & 1\end{array}\right]^{T}$ defines the horizontal layer of the interface in a Cartesian reference frame.

For a change from a porous to a homogeneous layer as shown in Fig. 3, there are seven equations defined for the boundary conditions. Normal and shear stresses have to correspond in both media at the interface $z_{T P M}=0$

$$
\hat{\mathbf{T}}_{T P M}^{z=0} \mathbf{n}-\hat{\mathbf{T}}_{H}^{z=d_{H}} \mathbf{n}=\mathbf{0},
$$

where the stresses in the mixture are defined as the sum of the stresses of the constituents.

In contrast to the solid, where all displacements are equated with the displacements of the homogeneous material, for the gas phase only the $z$-component is coupled:

$$
\begin{array}{r}
\hat{\mathbf{u}}_{S}(z=0)-\hat{\mathbf{u}}_{H}\left(z=d_{H}\right)=\mathbf{0} \\
\hat{u}_{G}^{z}(z=0)-\hat{u}_{H}^{z}\left(z=d_{H}\right)=0
\end{array}
$$

The height of the homogeneous layer is specified with $d_{H}$. In total seven equations are gained for this type of transition. The equations for the combination of other materials within compound absorbers can be derived analogously. A survey of the boundary-equations related to the different transitions depicted in table 1.

Table 1. Summary of the equations resulting out of the boundary conditions [3]

\begin{tabular}{cccc}
\hline & Stresses & Displacements & Eqns. \\
\hline Lamé-TPM & $\hat{\mathbf{T}}_{T P M}^{z=0} \mathbf{n}-\hat{\mathbf{T}}_{H}^{z=d_{H}} \mathbf{n}=\mathbf{0}$ & $\begin{array}{l}\hat{\mathbf{u}}_{S}(z=0)-\hat{\mathbf{u}}_{H}\left(z=d_{H}\right)=\mathbf{0} \\
\hat{u}_{G}^{z}(z=0)-\hat{u}_{H}^{z}\left(z=d_{H}\right)=0\end{array}$ & 7 \\
\hline \multirow{2}{*}{ Helmholtz-TPM } & $\hat{\mathbf{T}}_{T P M}^{z=0} \mathbf{n}+\hat{p}_{A}^{z=d_{A}} \mathbf{n}=\mathbf{0}$ & $\begin{array}{l}\hat{u}_{S}^{z}(z=0)-\hat{u}_{A}^{z}\left(z=d_{A}\right)=0 \\
\hat{u}_{G}^{z}(z=0)-\hat{u}_{A}^{z}\left(z=d_{A}\right)=0\end{array}$ & 5 \\
\hline \multirow{2}{*}{ Helmholtz-Lamé } & $\hat{\mathbf{T}}_{H}^{z=0} \mathbf{n}+\hat{p}_{A}^{z=d_{A}} \mathbf{n}=\mathbf{0}$ & $\hat{u}_{H}^{z}(z=0)-\hat{u}_{A}^{z}\left(z=d_{A}\right)=0$ & 4
\end{tabular}

In this ITM-based absorber model the number of unknowns, which is defined in equation (29) for arbitrary configurations of compound absorbers with $n_{i}$ layers of each material $i$ is reduced significantly compared to finite element approaches.

$$
n_{\text {tot }}=n_{T P M} \cdot 8+n_{H} \cdot 6+n_{A} \cdot 2-1
$$


In case of a single porous layer, which is presented in the examples in Chapter 5 the unknown coefficients of the fundamental system, which are stored in a vector $\mathbf{x}$, are calculated out of a linear equation system $\mathbf{K} \mathbf{x}=\mathbf{f}$ with only nine unknowns. The load, which is the pressure amplitude of the incident sound wave $p_{i}$ (compare figure 2), is defining the load vector $\mathbf{f}$ and the matrix $\mathbf{K}$ is obtained from the boundary conditions in table 1 as discussed in [3].

The wavenumber- and frequency-dependent impedance, which is used for the Lagrangian (12) and the virtual work of the non conservative forces (13) of the compound absorber in the equilibrium formulation (1), can be computed according to equation (30).

$$
Z\left(k_{x}, k_{y}, \Omega\right)=\frac{\hat{p}_{A}\left(k_{x}, k_{y}, \Omega\right)}{\hat{v}_{A}^{z}\left(k_{x}, k_{y}, \Omega\right)}
$$

\section{COUPLING THE SUBSYSTEMS AND ASSEMBLING THE EQUATION SYSTEM}

As Hamilton's principle is applied for the formulation of the equilibrium of the coupled system the Lagrangian has to be set up for all components of the problem and the virtual work must be computed for the external forces as well as for the dissipative behavior of the absorbers.

Normal and coupling modes, which are specified in the CMS approach in equation (4), are computed for the acoustic fluid as trial functions in the scope of a Ritz approach and the Lagrangian of the fluid as well as the virtual work of the external loads are computed with equations (2) and (3) respectively. The Lagrangian of the compound absorber and the virtual work of the non-conservative damping forces are computed with (12) and (13) for instance.

The unknown complex coefficients $\mathcal{A}_{i}$ and $\mathcal{B}_{i}$ refer to the normal and the coupling modes in the acoustic volume, whereas $\mathcal{C}_{i}$ are the coefficients of the trial functions of the compound absorber. The coupling condition of the fluid and the absorber at the interface, which is defined in equation (1) with the help of the vector of Lagrange multipliers $\lambda$, simply results in $\mathcal{B}_{i}=\mathcal{C}_{i}$ and $\overline{\mathcal{B}}_{i}=\overline{\mathcal{C}}_{i}$, if the same velocity pattern is chosen for the trial function of the absorber and for the boundary condition of the fluid at the absorber-interface. Thus the vectors for the unknown coefficients $\mathbf{x}$ and the corresponding conjugate complex values $\overline{\mathbf{x}}$ read:

$$
\begin{aligned}
& \mathbf{x}=\left[\begin{array}{ll}
\mathcal{A}_{1} \cdots \mathcal{A}_{m_{\max }} & \mathcal{B}_{1} \cdots \mathcal{B}_{n_{\max }}
\end{array}\right]^{T} \\
& \overline{\mathbf{x}}=\left[\begin{array}{ll}
\overline{\mathcal{A}}_{1} \cdots \overline{\mathcal{A}}_{m_{\max }} & \overline{\mathcal{B}}_{1} \cdots \overline{\mathcal{B}}_{n_{\max }}
\end{array}\right]^{T}
\end{aligned}
$$

The solution of the variational problem is reduced to a problem of minimization because of the Ritz approach. It is advantageous to express the conjugate complex coefficients with real and imaginary values in order to formulate the extremal problem:

$$
\begin{aligned}
& \mathbf{x}=\left[\mathbf{x}^{R}+i \mathbf{x}^{I}\right]^{T} \\
& \overline{\mathbf{x}}=\left[\mathbf{x}^{R}-i \mathbf{x}^{I}\right]^{T}
\end{aligned}
$$

The real and imaginary parts of the complex coefficients mark the new set of unknowns $\mathbf{y}=$ $\left[\begin{array}{ll}\mathbf{x}^{R} & \mathbf{x}^{I}\end{array}\right]^{T}$, where the total number of real valued unknowns is $2\left(m_{\max }+n_{\max }\right)$. Here $m_{\max }$ 
and $n_{\max }$ are the maximum numbers of normal and coupling modes respectively.

$$
\begin{aligned}
\mathbf{x}^{R} & =\left[\begin{array}{ll}
\mathcal{A}_{1}^{R} \cdots \mathcal{A}_{m_{\max }}^{R} & \mathcal{B}_{1}^{R} \cdots \mathcal{B}_{n_{\max }}^{R}
\end{array}\right]^{T} \\
\mathbf{x}^{I} & =\left[\begin{array}{ll}
\mathcal{A}_{1}^{I} \cdots \mathcal{A}_{m_{\max }}^{I} & \mathcal{B}_{1}^{I} \cdots \mathcal{B}_{n_{\max }}^{I}
\end{array}\right]^{T}
\end{aligned}
$$

For the consideration of the virtual work a vector $\delta \mathbf{y}$ is specified analogously. Carrying out the minimization of the Lagrangian one obtains a system of real valued linear equations

$$
\mathbf{K} \mathbf{y}=\mathbf{F}
$$

where the matrix of coefficients $K_{i j}$ reads

$$
K_{i j}=\frac{\partial^{2} \int_{0}^{T} L_{A} d t}{\partial y_{i} \partial y_{j}}+\frac{\partial^{2} \int_{0}^{T} L_{\mathrm{BC}}(Z) d t}{\partial y_{i} \partial y_{j}}+\frac{\partial^{2} \int_{0}^{T} \delta W_{\mathrm{BC}}^{\mathrm{nc}}(Z) d t}{\partial \delta y_{i} \partial y_{j}}
$$

and the load vector $\mathbf{F}=\left[\begin{array}{ll}\mathbf{F}^{R} & \mathbf{F}^{I}\end{array}\right]^{T}$ considers the external forces:

$$
F_{i}=-\frac{\partial \int_{0}^{T} \delta W_{\text {Load }}^{\mathrm{nc}} d t}{\partial \delta y_{i}}
$$

Defining submatrices $\mathbf{K}_{r s}$ for the matrix of coefficients, equation (34) reads:

$$
\left[\begin{array}{ll}
\mathbf{K}_{11} & \mathbf{K}_{12} \\
\mathbf{K}_{21} & \mathbf{K}_{22}
\end{array}\right]\left[\begin{array}{l}
\mathbf{x}^{R} \\
\mathbf{x}^{I}
\end{array}\right]=\left[\begin{array}{l}
\mathbf{F}^{R} \\
\mathbf{F}^{I}
\end{array}\right]
$$

In consequence of the complex property of the unknown coefficients the relations

$$
\begin{aligned}
& \mathbf{K}_{11}=\mathbf{K}_{22} \\
& \mathbf{K}_{21}=-\mathbf{K}_{12}
\end{aligned}
$$

hold for the submatrices.

\section{NUMERICAL EXAMPLES}

The application of the method is presented in the following examples.

\subsection{Application to a $1 d$ Structure}

In the first example the FSI of the acoustic fluid and porous absorbers is discussed in order to present the application of the method to measured impedances as well as to impedances out of numerical simulations. The rectangular acoustic volume, sketched in figure 4, with $V=\left[0, L_{x}\right] \times\left[0, L_{y}\right]=[0,3 \mathrm{~m}] \times[0,1 \mathrm{~m}]$ is modeled with Spectral Finite Elements according to [12]. 


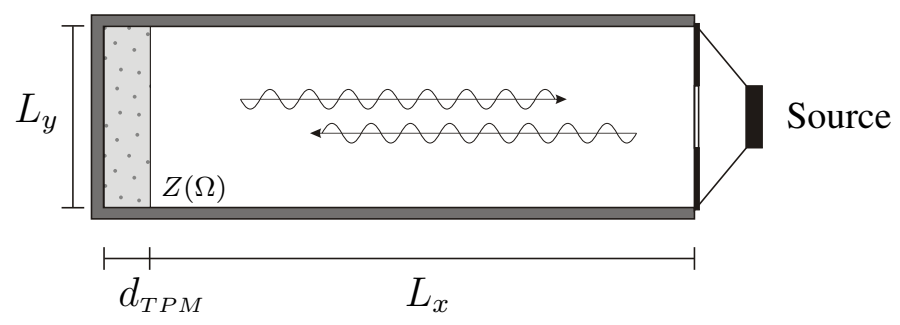

Figure 4. 1d structure with porous absorber

A layer out of Melamine Foam with a thickness of $7.2 \mathrm{~cm}$ is mounted on the reflective wall at $x=0$ and plane waves are excited in the system at $x=L_{x}$ in $x$-direction in the frequency range up to $275 \mathrm{~Hz}$. The response of the sound pressure within the cavity is evaluated along the $x$-coordinate. The computation is carried out with impedances, gained out of the numerical TPM-ITM-model, which is explained in section 3 and the results are compared with a computation based on impedances obtained from measurements. Figure 5 shows the transfer function for the pressure, averaged over the acoustic volume.

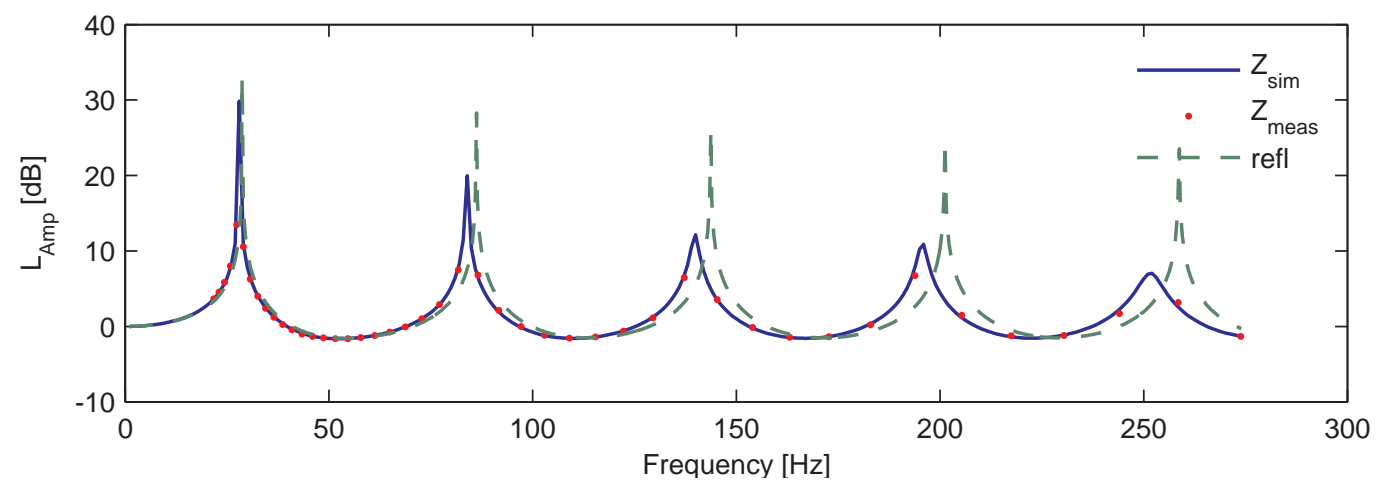

Figure 5. FSI-coupling with a layer of Melamine Foam

The results with the computed impedances $Z_{\text {sim }}$ are plotted against the solution with measured impedances $Z_{\text {meas }}$. A reduction of the amplitudes, especially at the location of the natural frequencies of the system, can be observed as well as the fact, that the porous absorber is working more efficiently for higher frequencies. Here the resulting wavelengths $\lambda$ are shorter and therefore the sound velocity within the absorber compared to the maximum velocity is higher than for low frequencies of excitation. The results also illustrate, that the simulation of the acoustic properties for porous foams with the TPM/ITM-model as a step of preprocessing for a FSI-simulation is suitable, because there is a good agreement between the results with the measured and the simulated impedances.

\subsection{Application to a $2 d$ Structure}

For arbitrary geometries the normal and coupling modes can be computed with the SFEM. The only restriction, given by the application of the ITM for the Lagrangian of the absorber, is, that the interface has to be plain. In the following a $2 \mathrm{~d}$ acoustic volume with an inclining rear-wall is considered. The wall containing the interface could be inclined as 
well. The geometry of the system is sketched in figure 6 , where $L_{x}^{1}=6 \mathrm{~m}, L_{x}^{2}=1.5 \mathrm{~m}$ and $L_{y}=2 \mathrm{~m}$. The model is set up with 192 spectral finite elements. The interface is covered with a $7.2 \mathrm{~cm}$ layer of Melamine Foam in analogy to the last example. A unit point source is located at $x=1.15 \mathrm{~m}$ and $y=0.77 \mathrm{~m}$. The location is chosen under the premise of exciting nearly all modeshapes.
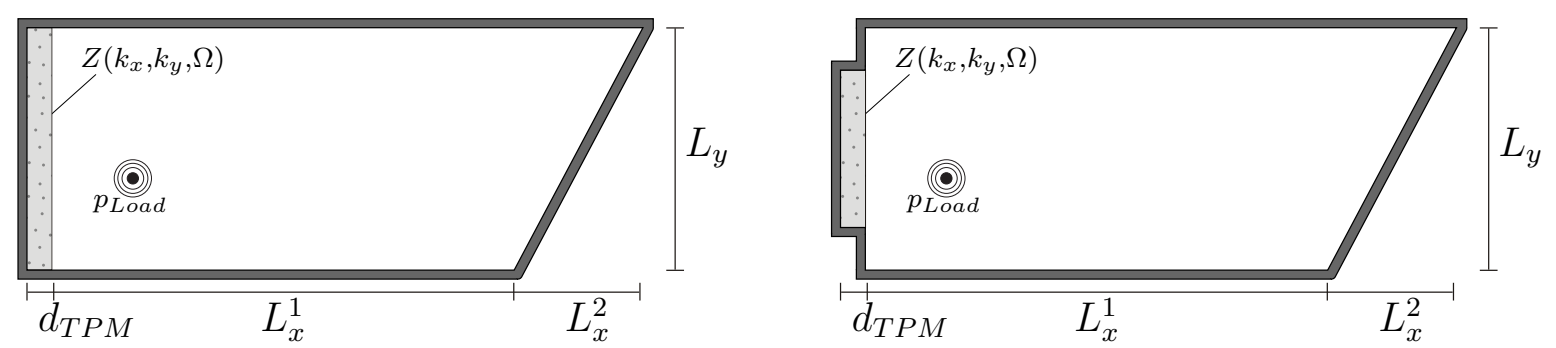

Figure 6. $2 \mathrm{~d}$ structure with with inclined wall and porous absorber (holohedral and subregional coupling)

In figure 8 the steady state response for the sound pressure level is sketched. The different interface-specifications are compared for a frequency of excitation of $163 \mathrm{~Hz}$. Due to the fact, that the frequency of excitation is near to a natural frequency, one specific modeshape is excited, which would lead to very high sound pressures in case of an undamped system (compare figure 7). A significant reduction is achieved due to the application of the absorptive layer at the boundary. Comparing both results in figure 8 one observes lower sound pressure levels for the holohedral case, because here the absorptive area, which is introduced into the system by the boundary condition, and therefore the dissipation of energy is higher than for subregional coupling.

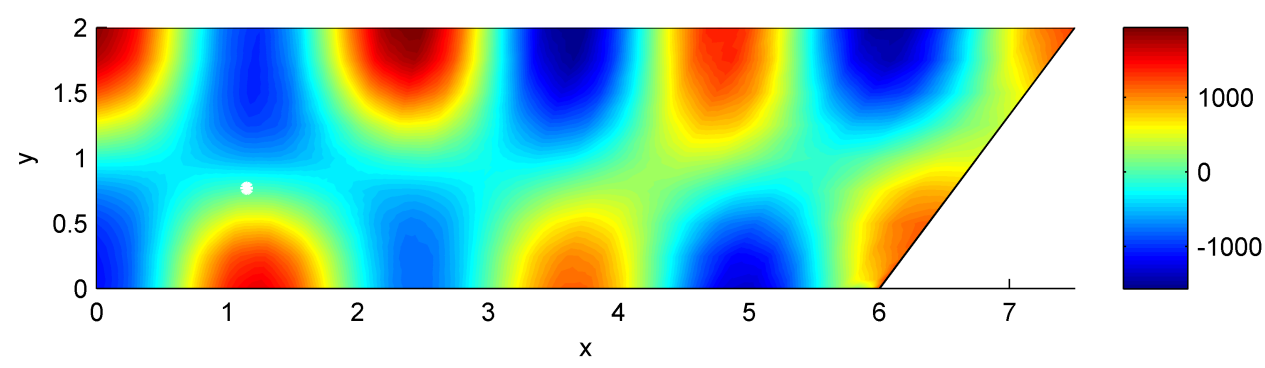

Figure 7. Sound-pressure $p(x, y)[\mathrm{Pa}]$ for a frequency of excitation of $163 \mathrm{~Hz}$ - spatial resolution for a unit point source at $x=1.15 \mathrm{~m}$ and $y=0.77 \mathrm{~m}$ - undamped system 

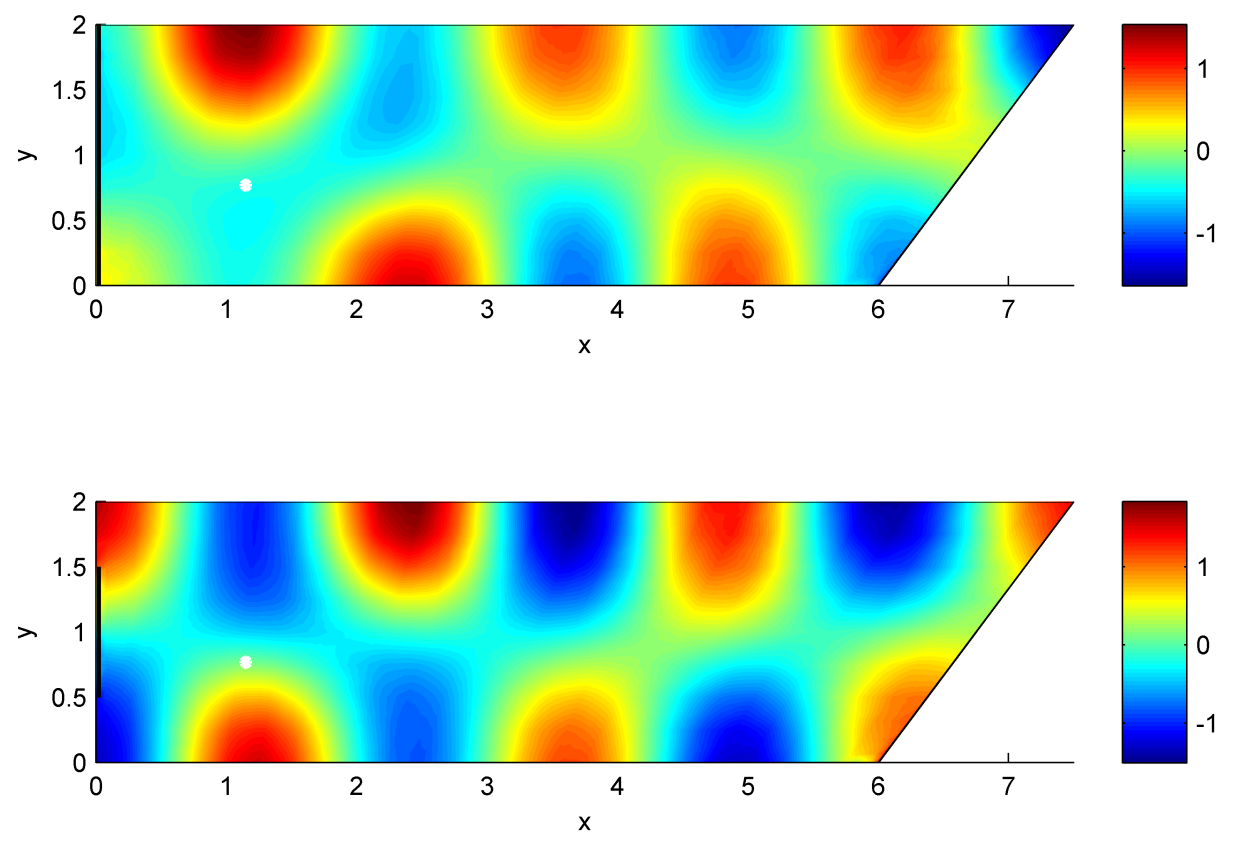

Figure 8. Sound-pressure $p(x, y)[\mathrm{Pa}]$ for a frequency of excitation of $163 \mathrm{~Hz}$ - spatial resolution for a unit point source at $x=1.15 \mathrm{~m}$ and $y=0.77 \mathrm{~m}$-comparison of holohedral and subregional coupling

\section{CONCLUSION}

In this contribution a method is presented to compute acoustic cavities under harmonically oscillating loads in order to get phase correct results with a spatial resolution for the sound field. The application of the CMS approach provides the possibility to recalculate the system for small changes in geometry or load data with low numerical effort. Changes in the location of boundary structures for example do not require a recalculation of the structure's normal modes or the impedances. The normal and constraint modes for the acoustic cavity are calculated with the SFEM. This method provides a spectral convergence for an increasing number of degrees of freedom related to the p-refinement. The SFEM formulation is implemented in order to model arbitrary geometries for the acoustic cavity. Because of the Fourier transforms in the scope of the ITM however the interface has to be plane. With the help of the TPM absorptive foam structures, used in acoustic design, can be modeled close to reality, which is verified in a comparison with measurement results in an impedance tube. Layered boundary structures as compound absorbers, consisting of homogeneous and porous materials, are modeled efficiently using the Fourier transform. Therefore the number of unknowns can be reduced significantly compared to fully discretized coupled systems. Due to the configuration of the method, especially due to the combination of single subsystem results, the sensitivity of the final results for the coupled system to changes of subsystem parameters can be estimated more efficiently than in a total approach. This is advantageous for engineering design processes. The application of the derived FSI method is presented for a $1 \mathrm{~d}$ benchmark case and a $2 \mathrm{~d}$ geometry. The results show, that this approach is very promising for com- 
putation of more complex problems like optimization processes, uncertainty calculations or computations in a higher frequency range with averaging in the post processing.

\section{References}

[1] de Boer, R.: Theory of Porous Media. Highlights in Historical Development and Current State. Springer (2000)

[2] de Boer, R.: Trends in Continuum Mechanics of Porous Media. Theory and Applications of Transport in Porous Media. Springer (2005)

[3] Buchschmid, M.: ITM-based FSI-models for rooms with absorptive boundaries. Ph.D. thesis, TU München (2011)

[4] Buchschmid, M., Müller, G.: Modeling of wave number dependent absorptive characteristics with the help of the theory of porous media. In: EURODYN Conference (2008)

[5] Buchschmid, M., Müller, G., Kropp, A.: Closing the mid-frequency-gap: Acoustic biw design using a hybrid sea-fea approach. In: The 16th International Congress on Sound and Vibration, Krakow, Poland, ICSV16 (2009)

[6] Buchschmid, M., Pospiech, M., Müller, G.: ITM-based FSI-models for applications in room acoustics. In: COMPDYN Conference (2009)

[7] Buchschmid, M., Pospiech, M., Müller, G.: Coupling impedance boundary conditions for absorptive structures with spectral finite elements in room acoustical simulations. Computing and Visualization in Science pp. DOI: 10.1007/s00,791-010-0148-y (2011)

[8] Gekeler, E.: Mathematische Methoden zur Mechanik. Springer (2006)

[9] Hurty, W.: Vibrations of structural systems by component-mode synthesis. Journal of Engineering Mechanics (ASCE) 86(4), 51-69 (1960)

[10] Hurty, W.: Dynamic analysis of structural systems using component modes. AIAA Journal 3 (4), 678-685 (1965)

[11] Larbi, W., De, J.F., Ohayon, R.: Finite element formulations for structural-acoustic internal problems with poroelastic treatment. In: COMPDYN Conference (2009)

[12] Pospiech, M.: Numerical simulations in room acoustics using direct coupling techniques and finite elements. Ph.D. thesis, TU München (2011) 